\title{
Grounding the figure: Surface attachment influences figure-ground organization
}

\author{
SHAUN P. VECERA \\ University of Iowa, Iowa City, Iowa \\ and \\ STEPHEN E. PALMER \\ University of California, Berkeley, California
}

\begin{abstract}
We investigated whether the lower region effect on figure-ground organization (Vecera, Vogel, \& Woodman, 2002) would generalize to contextual depth planes in vertical orientations, as is predicted by a theoretical analysis based on the ecological statistics of edges arising from objects that are attached to surfaces of support. Observers viewed left/right ambiguous figure-ground displays that occluded middle sections of four types of contextual inducers: two types of attached, receding, vertical planes (walls) that used linear perspective and/or texture gradients to induce perceived depth and two types of similar trapezoidal control figures that used either uniform color or random texture to reduce or eliminate perceived depth. The results showed a reliable bias toward seeing as "figure" the side of the figure-ground display that was attached to the receding depth plane, but no such bias for the corresponding side in either of the control conditions. The results are interpreted as being consistent with the attachment hypothesis that the lower region cue to figure-ground organization results from ecological biases in edge interpretation that arise when objects are attached to supporting surfaces in the terrestrial gravitational field.
\end{abstract}

In optical projections of multiobject visual scenes, objects often partially occlude other objects, producing adjacent image regions that share a contour. To determine the shapes and relative distances of corresponding environmental objects, the visual system must determine which regions correspond to occluding foreground surfaces and which to occluded background surfaces. Figure-ground processes are assumed to be responsible for labeling regions as occluding figures or occluded grounds - in part, to determine which regions should be attended and recognized from the shape of the contour on that side.

Gestalt psychologists approached figure-ground organization by identifying perceptual cues that influence which region(s) are likely to appear as figure versus ground (for reviews, see Palmer, 1999, 2002). Rubin (1915/1958) first identified and studied several basic figural cues, such as the fact that a smaller region tends to be perceived as fig-

This research was supported in part by Grants BCS 99-10727 and BCS 03-39171 from the National Science Foundation, awarded to the first author. We thank Mike Braunstein and two anonymous reviewers for their comments. The order of authorship is equal. Correspondence should be addressed either to S. P. Vecera, Department of Psychology, University of Iowa, E11 Seashore Hall, Iowa City, IA 52242-1407 or to S. E. Palmer, Department of Psychology, University of California, Berkeley, CA 94720-1650 (e-mail: shaun-vecera@uiowa.edu or palmer@cogsci .berkeley.edu). ure and a larger region as ground. Similarly, symmetric regions (Bahnsen, 1928), convex regions (Kanizsa \& Gerbino, 1976), familiar regions (Peterson, 1994, 1999), and regions with wide bases (Hulleman \& Humphreys, 2004) are more likely to be perceived as figure than are asymmetric, concave, unfamiliar, and narrow-based regions, respectively. Of most relevance to the present article, a region appearing below a common edge in the visual field is more likely to be perceived as figure than is a region appearing above this edge (Vecera, Vogel, \& Woodman, 2002), as is illustrated in Figure 1 A.

Although certain aspects of figure-ground cues are well understood, their origin is not. Some writers have suggested that perceptual organization processes might be innate (e.g., Koffka, 1935; Zuckerman \& Rock, 1957), whereas others have argued that they are learned through experience with the visual environment (Ross-Sheehy, Oakes, \& Vecera, 2003). In either case, environmental regularities are crucial, because statistical differences in the projected properties of closer versus farther objects are presumed to underlie the utility of such cues. Symmetric regions, for example, are more likely to be perceived as figures because symmetric regions in images are more likely due to the projection of a symmetric object (figure) than to two asymmetric objects accidentally creating a symmetric space between them (ground). Recent studies of the statistics of natural scenes document the eco- 

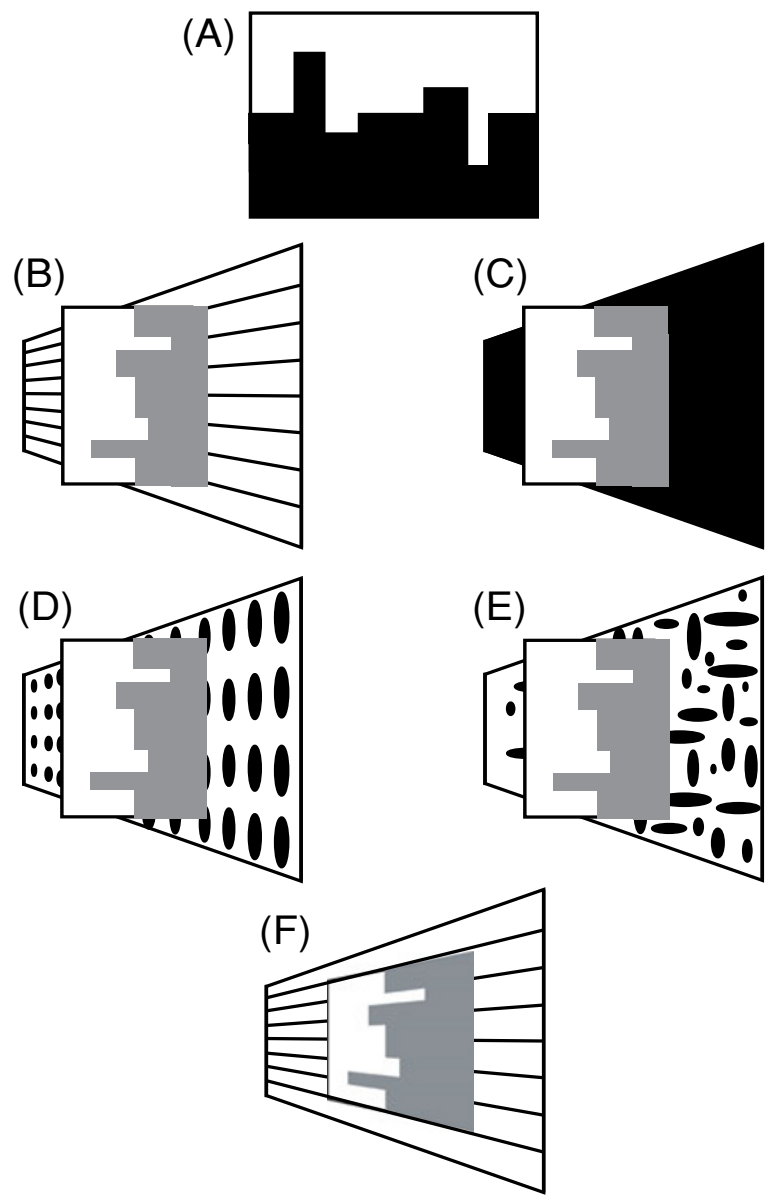

Figure 1. (A) The lower region cue, in which the lower (black) region appears as figure. (B) Depth plane stimuli used in the converging lines condition, (C) the solid trapezoid condition, (D) the oval gradient condition, and (E) the oval control condition. (F) A display in which figure-ground display is coplanar with depth plane (see the text for a discussion).

logical validity of certain locally defined figure-ground cues, including smaller size, convexity, and, of particular relevance to the present article, lower region (Fowlkes, Martin, \& Malik, 2001).

One premise of the present research is that the statistical regularities of the environment that influence figure-ground organization are not restricted to intrinsic properties of individual objects. Global scene structure may also affect figure-ground organization, as with the figure-ground cue of lower region depicted in Figure 1A (Vecera et al., 2002). When two regions are located above and below a shared contour that is roughly horizontal on a global scale, the lower of the two regions tends to be perceived as figure (Vecera et al., 2002). Here, we propose and explore a possible reason for the lower region preference that is based on the fact that objects are generally attached to supporting surfaces below them in the terrestrial gravitational field. The predominant supporting surface is, of course, the ground plane (Gibson, 1950), and many perceptual processes are influenced by contact with a ground surface (Bian, Braunstein, \& Andersen, 2005; McCarley \& He, 2000; Meng \& Sedgwick, 2001). Even in the absence of an explicit ground plane, the visual system assumes that objects are supported by such a surface (Ooi, Wu, \& He, 2001; Philbeck \& Loomis, 1997).

Our argument for the role of attachment is as follows. An optically projected edge can arise from two different surfaces due to either changes in depth (depth edges) or changes in surface orientation (orientation edges). Depth edges are the most relevant for figure-ground organization because they result from the surface on one side's being closer to the observer than is the surface on the other side, with a depth discontinuity between them (e.g., a figure in front of a background). A bias toward the lower region's being perceived as figure is ecologically supported if the depth statistics at globally horizontal depth edges indicate that the lower region is closer to the observer more often than the upper region is. We suggest that the lower region bias arises, in part, from the fact that most objects are attached to supporting surfaces beneath them.

To see why, consider first a gravitation-free world in which opaque, convex objects float at random positions and are viewed against a uniform, distant, background surface (e.g., the sky, a wall, the ground, etc.). Figure 2A depicts an opaque, convex object whose edges are labeled either as depth edges, identified by $\mathrm{C} / \mathrm{F}$ labels indicating the closer $(\mathrm{C})$ and farther $(\mathrm{F})$ surfaces, or as orientation edges, indicated by $\mathrm{O} / \mathrm{O}$ labels. All else being equal, there is no clear ecological basis for a lower region bias in such a world, because there are equal numbers of depth edges in which the lower region is closer (at the tops of objects) and in which the upper region is closer (at the bottoms of objects). For analogous reasons, there is no ecological basis for a bias toward either the left region or the right region of depth edges being seen as closer to the observer in this gravitation-free world.

Now consider a gravitational world such as our own, in which the same objects are supported by surfaces beneath them (Figure 2B). Here, the horizontal depth edges at the bottoms of objects have become orientation edges because the object surface meets the supporting surface at the edge, so that neither region is closer. As a result, the depth statistics at roughly horizontal edges become strongly biased toward the lower region's being closer and, thus, support a lower region heuristic in figure-ground perception. In contrast, the depth statistics of vertical edges will still be unbiased when support comes primarily from attachment to lower surfaces, because vertical depth edges are still equally distributed between cases in which the left and the right regions are closer.

Note that the analysis thus far is largely viewpoint independent; the lower region bias results from geometrical regularities in gravitationally structured scenes themselves. It does assume that the observers' heads are roughly upright in the gravitational field and that roughly horizontal edges in the image tend to be projections of roughly horizontal edges in the environment, but both assumptions seem 
(A)

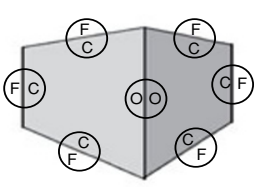

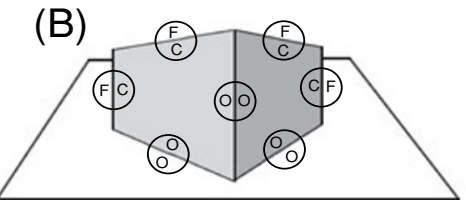

(C)

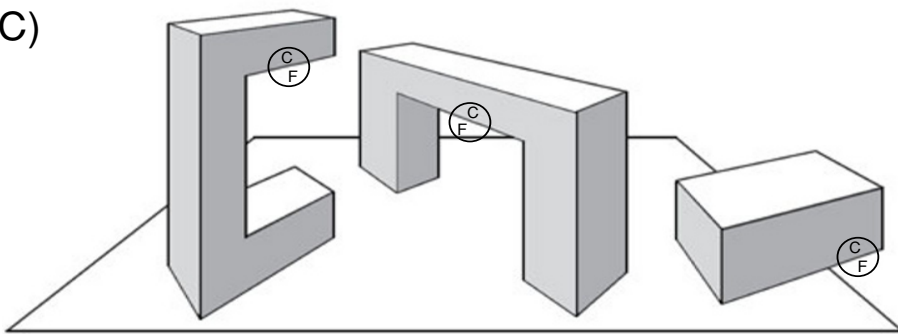

(D)

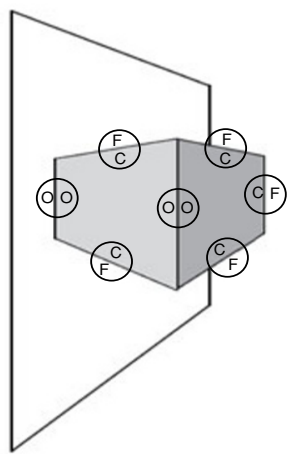

(E)

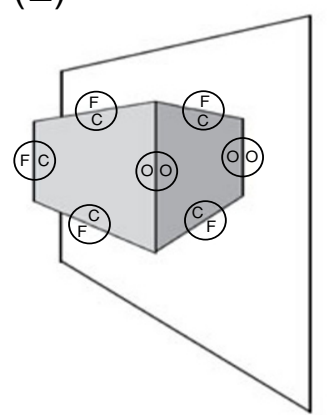

Figure 2. Illustrations of the ecological basis of the attachment hypothesis. (A) In a gravitation-free world of floating objects, there is equal evidence for lower and upper regions being closer. (B) In a gravitational world of objects attached to supporting surfaces below them, evidence for lower regions being closer is increased. (C) Complex objects with concavities and only partial support from below produce situations in which upper regions can be closer. (D) When objects are attached to vertical surfaces of support on the left, left regions are more likely to be closer. (E) When objects are attached to vertical surfaces of support on the right, right regions are more likely to be closer (see the text for additional details).

statistically reasonable. Nevertheless, we acknowledge that there are other factors that complicate the analysis we have just given. For example, complex objects with deep concavities can often be parsed into multiple convex parts that are attached to each other, as Hoffman and Richards (1984) have argued, and not all such convex parts need to be independently supported from below. As the C-shaped and arch-shaped objects in Figure 2C illustrate, parts that are not supported by surfaces directly below them can give rise to roughly horizontal depth edges in which the upper region is closer to the observer. Partial support by a lower surface also produces situations of overhang in which the upper region is closer to the observer (Figure 2C). Even so, the high probabilities of upright viewing and of full attachment to supporting lower surfaces suggest that the lower region principle should be useful in figure-ground perception. Further complications arise in multiobject dis- plays because closer objects often occlude parts of farther objects, but here again, gravitational constraints favor the visibility of upper edges over lower edges, so it is unlikely that occlusion will do anything but amplify the bias produced by attachment.

If attachment to lower supporting surfaces is at least part of the reason for the lower region bias in figural assignment, analogous biases should be introduced when objects are perceived as attached to vertical depth planes. As is illustrated in Figures 2D and 2E, there should be a left-region bias in figure-ground perception for vertical edges of objects that are attached to surfaces on the left side relative to the observer and a right-region bias in figure-ground perception for vertical edges of objects that are attached to surfaces on the right side relative to the observer. The present experiment provides an initial test of this prediction based on the attachment hypothesis. 
Ambiguous left/right figure-ground displays were placed in front of and partly occluding vertical, receding depth planes (or walls), so that either the left or the right edge of the figure-ground display (but not both) appeared to be attached to this surface (Figures 1B and 1D). The figure-ground display contained a red region and a green region that were equal in area, convexity, familiarity, and all other known figure-ground cues, and observers were asked to report which region, red or green, appeared to be the foreground figure. Prior results have repeatedly shown that such displays produce no measurable figural preference (Vecera, 2004; Vecera et al., 2002). If depth perception induced by attachment to a receding depth plane affects figure-ground assignment, as the attachment hypothesis predicts, the depth planes should bias the attached region toward being seen as figural. Importantly, because of the juxtaposition between the depth plane and the figure-ground display, both regions of the figureground display appear closer than the depth plane and appear figurelike. Thus, any effect of perceiving the near (attached) region of the figure-ground display as more figurelike would need to overcome the general percept of perceiving the entire figure-ground display as a single, foreground stimulus.

An inspection of Figures 1B and 1D suggests a confounding factor, however: There is simply more visible area on the near side of the depth plane than on the far side. A figure-ground preference for the attached region might, therefore, arise from attention's being drawn to the larger side of the inducing depth plane. Vecera, Flevaris, and Filapek (2004) have shown that stimulus-driven attention to a region can influence figure-ground assignment, so if attention were drawn to the larger, wide end of the depth plane by the existence of more visual information on that side, attention could spill over to the adjacent figure-ground portion of display and bias figure-ground assignment toward the region attached to the larger part of the inducing figure.

To rule out this alternative, we created two control displays (Figures 1C and 1E), in which the depth cues of linear perspective and/or texture gradients were greatly reduced. If the attachment hypothesis is correct and if the weakening of linear perspective and texture cues flattens perception of the depth plane to a trapezoid perpendicular to the line of sight, no figural bias should be evident in the control displays, because the figure-ground display is perceived to be coplanar with the trapezoid. If figureground organization is affected by attentional differences that are due to size or other nondepth information, however, regions at the larger end of the control context should still tend to be perceived as "figure" in the control displays more often than regions at the smaller end.

\section{METHOD}

\section{Participants}

The 20 participants in each condition were University of Iowa undergraduates who were naive about the purpose of the study, had normal or corrected-to-normal vision, and received course credit for their participation.

\section{Stimuli}

Figure-ground displays similar to those in previous studies (Vecera et al., 2002) were viewed from a distance of $70 \mathrm{~cm}$. They contained two regions, one red and one green, that shared an irregular, vertical contour, so that the two regions had equal area and convexity and neither was familiar in shape (Figures 1B-1E). There were four different contours. The number of displays was doubled by also using the mirror image of each contour, and there were two versions of each of these eight contours, corresponding to the red/ green color combinations (red on the left and red on the right). Thus, there were a total of 16 figure-ground displays, each measuring $4.7^{\circ}$ tall $\times 3.6^{\circ}$ wide. The red/green color values were those used in previous studies (see Vecera et al., 2002).

The bounding contours of the depth plane inducers consisted of black lines on a white background and measured $11.0^{\circ}$ at the wide (near) end and $2.2^{\circ}$ at the narrow (distant) end. Examples of the depth planes appear in Figures 1B-1E. The depth plane was $6.7^{\circ}$ long. In the converging lines condition (Figure 1B), the depth plane was filled with seven lines that converged toward a vanishing point at the intersection of the two long sides of the depth plane; the lines were separated by $0.8^{\circ}$ at the near end of the depth plane and by $0.3^{\circ}$ at the distant end of the depth plane. In the solid trapezoid condition, the outline frame of the depth plane was colored black. In the oval gradient condition, the depth plane was filled with ovals that diminished regularly in size with depth. In the oval control condition, the same ovals filled the trapezoidal outline but were randomly positioned within the depth plane. In the latter two displays, there were a total of 32 ovals, the largest measuring $0.3^{\circ}$ wide $\times 1.2^{\circ}$ tall, and the smallest measuring $0.16^{\circ}$ wide $\times 0.25^{\circ}$ tall.

The red/green figure-ground displays were placed on the depth plane inducers as shown in Figure 1. The nearer/larger end of the depth plane was adjacent to the red region in half of the displays and the green region in the other half.

\section{Procedure}

The participants were instructed to report the color of the region that appeared to be the foreground figure. Prior to testing, each participant was shown Rubin's (1915/1958) face-vase figure to illustrate the phenomenon of figure-ground assignment. The participants were told that either the faces or the vase, but not both, could be perceived as lying in the foreground and would appear to be closer than the other region. The participants were asked to try to perceive both the faces and the vase as figure in alternation.

Each trial began with a $500-\mathrm{msec}$ fixation cross. Next, the figure-ground display and depth plane inducer were presented simultaneously until the observer reported which region, red or green, appeared as the foreground figure. Responses were made using a response box with a red key on the left and a green key on the right. Each observer received 32 total trials in a single block.

\section{RESULTS}

The results, as plotted in Figure 3, show that the observers reported perceiving the region attached to the contextual depth plane as the "figure" at above-chance rates when the contextual object contained strong and consistent depth information (Figures 1B and 1D). When the contextual inducer contained weaker depth information, however, no figural biases were evident for the regions adjacent to the larger side of the context. A one-factor ANOVA indicated significant differences among some of the conditions $[F(3,76)=5.9, p<.005]$. Further analyses compared each condition against chance $(50 \%)$ to reveal any preference in perceiving one region as figure. In the converging line displays, the observers reported perceiving the region 


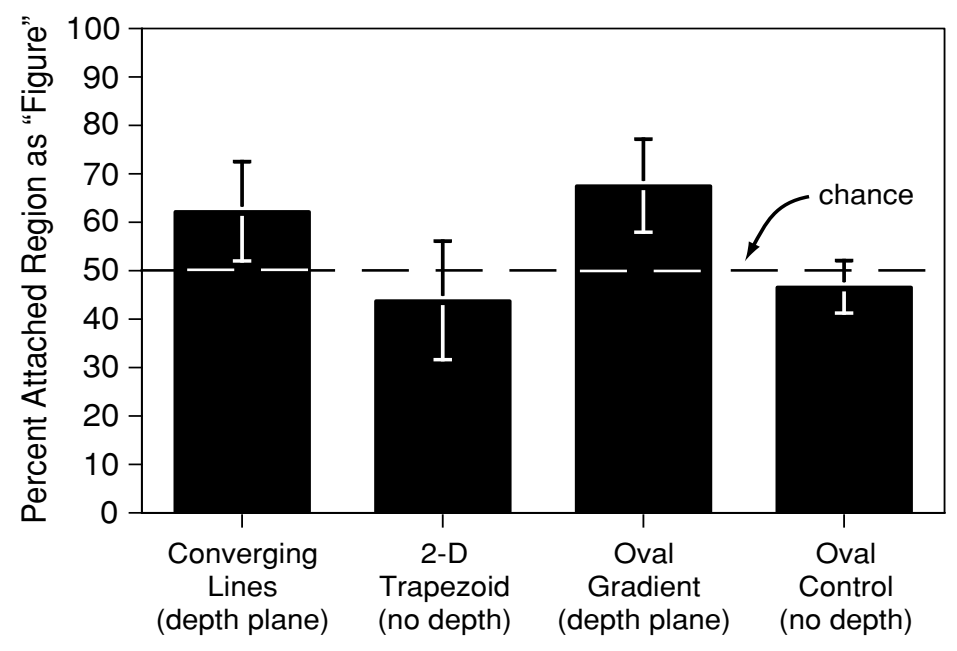

Figure 3. Percentage of trials on which the region attached to the contextual depth inducer was perceived as figure in the four experimental conditions. Significant biases were observed for the converging line and oval gradient displays, in which the depth planes were perceived as surfaces oriented in depth. When otherwise similar contexts did not carry depth information (in the solid trapezoid and oval control displays), there were no significant contextual effects. Error bars show 95\% confidence intervals on comparisons against chance.

adjacent to the nearer side of the depth plane as the "figure" on $61.9 \%$ of the trials, significantly above chance $(50 \%)$ $[t(19)=2.4, p<.03]$. However, no such figural bias was evident in the solid trapezoid displays $(43.5 \%)[t(19)=$ $1.1, p>.30]$, in which the same figure-ground displays were placed on a surface that was more readily perceived as a flat two-dimensional trapezoid. In the oval gradient displays, the observers reported perceiving the region attached to the depth plane as the "figure" on $67.2 \%$ of the trials, which was significantly above chance $[t(19)=3.7$, $p<.005]$. This bias was eliminated, however, in the oval control displays $(46.3 \%)[t(19)=1.4, p>.15]$.

Similar results arise from between-condition analyses. Collapsing across the different types of depth inducers and non-depth inducers shown in Figure 1, we find that the figure-ground bias is significantly greater when the inducer is a surface receding in depth (in the combined converging lines and oval gradient displays) than when it is not (in the combined solid trapezoid and oval control displays) $[t(78)=4.2, p<.0001]$.

The method we have used to assess figural status is admittedly subjective, in keeping with the majority of work on figure-ground organization. The robustness of the present results could be further investigated using more objective methods, such as a shape memory task (e.g., Driver \& Baylis, 1996; Vecera et al., 2004) or trading relations with binocular disparity (Burge, Peterson, \& Palmer, 2005).

\section{DISCUSSION}

The present results demonstrate that perceived attachment to contextual depth planes can systematically influence figure-ground organization: Regions seen as attached to a surface receding in depth are more likely to be perceived as figure than is the other side of an otherwise ambiguous figure-ground display. This bias depends on the nearby surface's being perceived as receding in depth, because manipulations that weaken or eliminate its perceived slant abolish the effect. Our results thus indicate that the environmental regularities that affect figure-ground organization are not limited to regularities of individual shapes or regions but extend to more global scene-based regularities.

Precisely why depth relations between otherwise ambiguous figure-ground displays and attached depth planes produce this bias is less clear. Our preferred interpretation is that the receding depth plane induces the region located closer to its nearer end to be seen as attached to the plane, which then triggers the attachment bias to see that region as closer and figural. There is another depth-related explanation that has not yet been ruled out, however: Attention may be drawn more strongly to the closer end of the contextual depth plane simply because it is nearer to the observer, and this additional attention might spill over to the adjacent region of the figure-ground display.

One way to differentiate these two accounts is shown in Figure $1 \mathrm{~F}$, where the ambiguous figure-ground display is presented as coplanar with the receding depth plane. ${ }^{1}$ The attentional account predicts that there would be an even stronger bias to see the closer half of the display as figural, because the figure-ground display itself is now slanted in depth and, therefore, subject to the hypothesized attentional bias toward closer objects. The attachment hypothesis, however, predicts that the contextual depth plane effect will be diminished or eliminated under these circumstances because the entire figure-ground display is 
now perceived as coplanar with the receding depth plane. Under such circumstances, both sides are perceived as attached to the plane, so the attachment bias simply does not apply. The results from 6 observers suggest that there is no figural bias in such displays. These observers reported perceiving the closer (gray) region as figure on $50 \%$ of the trials and reported being able to alternate between perceiving either region as figure. These results suggest that an attentional account in terms of a bias toward closer regions is unlikely to be correct.

Assuming that the attachment hypothesis provides a good explanation of the present data, what are its implications for understanding the lower region effect? One possibility is that the attachment hypothesis alone is sufficient to explain it. Another is that retinal orientation alone can explain it. Why retinal orientation should matter-other than because of heuristics derived from depth-related regularities (see below) - is unclear, however. The retinal hypothesis also fails to provide an explanation of the present results. A third possibility is that both factors are at work. Unfortunately, the present data do not discriminate among these hypotheses.

One way of differentiating between depth and retinal orientation accounts of the lower region effect is in terms of egocentric versus environmental frames of reference. If the lower region cue is based on attachment to lower supporting surfaces, it is reasonable to predict that the lower region effect would be governed by gravitational and/or environmental reference frames, rather than by observers' egocentric (viewer-centered) retinal reference frames, because objects are supported by surfaces that are gravitationally below them even when the observer's head is tilted so that some other direction is retinally downward. Using similar logic, Rock (1973) investigated the perceived and remembered shapes of novel objects as viewed by observers whose heads were tilted and discovered that shape perception is based primarily on environmental and/or gravitational reference frames. If the attachment hypothesis underlies the lower region effect, it is plausible to expect that the lower region effect will likewise be governed by an environmental reference frame, so that the environmentally and/or gravitationally lower region will be biased to be seen as closer and figural. If the lower region effect is due to retinal orientation, however, it should be governed by an egocentric, retinal reference frame, so that the retinally lower region will be seen as closer and figural.

Vecera (2004) recently performed just such an experiment and found that the lower region cue is clearly based on viewer-centered retinal coordinates. Observers viewed lower region displays with their heads upright, tilted $90^{\circ}$, or upside-down, and the results unequivocally showed that the lower region preference was determined by the observer's retinal orientation. These data thus appear to support the retinal orientation hypothesis of the lower region effect, rather than the attachment hypothesis. Given our stated preference for the attachment account, it is therefore incumbent on us to reconcile the implicit conflicts among Vecera's (2004) findings, Rock's (1973) results, and the present data.

Our proposed solution is based on three interrelated hypotheses: (1) The environmental depth regularities that we believe underlie both the lower region effect and the present depth plane effect can be heuristically approximated in terms of viewer-centered retinal reference frames, because the head is usually roughly upright when an observer is looking at the environment; (2) the determination of figure versus ground occurs relatively early in processing, before orientation constancy is complete, and is, therefore, governed by retinal reference frames; and (3) the explicit representation of object shape occurs after orientation constancy has been achieved and is, therefore, governed by the gravitational/environmental reference frame. These three hypotheses together provide a plausible account for why the lower region might be governed by a retinal reference frame and still be based on environmental depth considerations: Figure-ground relations are determined before true environmental information is available, so that preconstancy retinal orientations are all that is available as a depth-related heuristic. Because the head is generally upright during the vast majority of one's visual processing, however, the correlation between retinal and environmental orientation would be high enough to be useful for figure-ground organization. The same framework also explains why Rock's (1973) shape memory effects differ from the lower region effect in figure-ground organization: Explicit shape descriptions are computed after orientation constancy occurs, whereas figure-ground organization is computed before. Although our account is admittedly more complex than the simple retinal orientation hypothesis of the lower region, it has several desirable features. First, it explains both the lower region effect and the present depth plane effect within the same, coherent, ecologically based theoretical framework. Second, it provides an explanation of why lower region effects are governed by retinal orientation - namely, because they are caused by a depth-based heuristic that depends on the high correlation between retinal and environmental orientations under standard viewing conditions. Third, it integrates both of these effects in an interesting way with the seemingly contradictory results obtained by Rock showing that environmental reference frames are used in shape perception.

Our account does make the clear and testable prediction that other orientation-sensitive factors in figure-ground organization, such as region orientation and symmetry, should also be defined in viewer-centered, retinal coordinates, rather than in environmentally centered, gravitational coordinates. We are currently testing these predictions. If confirmed, they would provide fascinating dissociations between the conscious phenomena of shape perception and the presumably preconscious processes that underlie figure-ground organization. We already know that conscious perception of and memory for both object symmetry (Rock \& Leaman, 1963) and object orientation (Rock, 1973; Wiser, 1981) are largely governed 
by environmental reference frames. It would, therefore, be both surprising and revealing if these very same factors operated in figure-ground organization within retinally defined, viewer-centered reference frames that operate outside of visual awareness.

\section{REFERENCES}

BAHNSEN, P. (1928). Eine Untersuchung über Symmetrie und Asymmetrie bei visuellen Wahrnehmungen. Zeitschrift für Psychologie, 108, 129-154.

Bian, Z., Braunstein, M. L., \& Andersen, G. J. (2005). The ground dominance effect in the perception of 3-D layout. Perception \& Psychophysics, 67, 802-815.

Burge, J., Peterson, M. A., \& Palmer, S. E. (2005). Ordinal configural cues combine with metric disparity in depth perception. Journal of Vision, 5, 534-542. Available at journalofvision.org/5/6/5/, doi:10.1167/5.6.5.

Driver, J., \& BAYLIS, G. C. (1996). Edge-assignment and figure-ground segmentation in short-term visual matching. Cognitive Psychology, 31, 248-306.

Fowlkes, C., Martin, D., \& Malik, J. (2001). Understanding Gestalt cues and ecological statistics using a database of human segmented images. Paper presented at the Third Workshop on Perceptual Organization in Computer Vision, Vancouver, BC.

GiBson, J. J. (1950). The perception of the visual world. Boston: Houghton Mifflin.

Hoffman, D. D., \& Richards, W. A. (1984). Parts of recognition. Cognition, 18, 65-96.

Hulleman, J., \& Humphreys, G. W. (2004). A new cue to figure-ground coding: Top-bottom polarity. Vision Research, 44, 2779-2791.

Kanizsa, G., \& Gerbino, W. (1976). Convexity and symmetry in figure-ground organization. In M. Henle (Ed.), Vision and artifact (pp. 25-32). New York: Springer.

KoffKa, K. (1935). Principles of gestalt psychology. New York: Harcourt Brace.

McCarley, J. S., \& He, Z. J. (2000). Asymmetry in 3-D perceptual organization: Ground-like surface superior to ceiling-like surface. Perception \& Psychophysics, 62, 540-549.

Meng, J. C., \& SeDGWICK, H. A. (2001). Distance perception mediated through nested contact relations among surfaces. Perception \& Psychophysics, 63, 1-15.

OoI, T. L., Wu, B., \& He, Z. J. (2001). Distance determined by the angular declination below the horizon. Nature, 414, 197-200.

Palmer, S. E. (1999). Vision science: Photons to phenomenology. Cambridge, MA: MIT Press.
Palmer, S. E. (2002). Perceptual organization in vision. In H. Pashler \& S. Yantis (Eds.), Stevens' handbook of experimental psychology: Vol. 1. Sensation and perception (pp. 177-234). New York: Wiley.

Peterson, M. A. (1994). Object recognition processes can and do operate before figure-ground organization. Current Directions in Psychological Science, 3, 105-111.

Peterson, M. A. (1999). What's in a stage name? Comment on Vecera and O'Reilly (1998). Journal of Experimental Psychology: Human Perception \& Performance, 25, 276-286.

Philbeck, J. W., \& Loomis, J. M. (1997). Comparison of two indicators of perceived egocentric distance under full-cue and reduced-cue conditions. Journal of Experimental Psychology: Human Perception \& Performance, 23, 72-85.

Rock, I. (1973). Orientation and form. New York: Academic Press.

Rock, I., \& LEAMAN, R. (1963). An experimental analysis of visual symmetry. Acta Psychologica, 21, 171-183.

Ross-Sheehy, S. M., OAKes, L. M., \& Vecera, S. P. (2003, October). Figure-ground segregation in human infants: Sitters versus non-sitters. Poster presented at the 2003 Biennial Meeting of the Society for Research in Child Development, Tampa, FL.

Rubin, E. (1958). Figure and ground. In D. C. Beardslee \& M. Wertheimer (Eds.), Readings in perception (pp. 194-203). Princeton, NJ: Van Nostrand. (Original work published 1915)

VECERA, S. P. (2004). The reference frame of figure-ground assignment. Psychonomic Bulletin \& Review, 11, 909-915.

Vecera, S. P., Flevaris, A. V., \& Filapek, J. C. (2004). Exogenous spatial attention influences figure-ground assignment. Psychological Science, 15, 20-26.

Vecera, S. P., Vogel, E. K., \& Woodman, G. F. (2002). Lower region: A new cue for figure-ground assignment. Journal of Experimental Psychology: General, 131, 194-205.

WISER, M. (1981, August). The role of intrinsic axes in shape recognition. Paper presented at the Third Annual Conference of the Cognitive Science Society, Berkeley, CA.

Zuckerman, C. B., \& Rock, I. (1957). A reappraisal of the roles of past experience and innate organizing processes in visual perception. Psychological Bulletin, 54, 269-296.

\section{NOTE}

1. We thank Mike Braunstein for suggesting this condition in his review of a previous version of the manuscript.

(Manuscript received March 31, 2005; revision accepted for publication December 12, 2005.) 